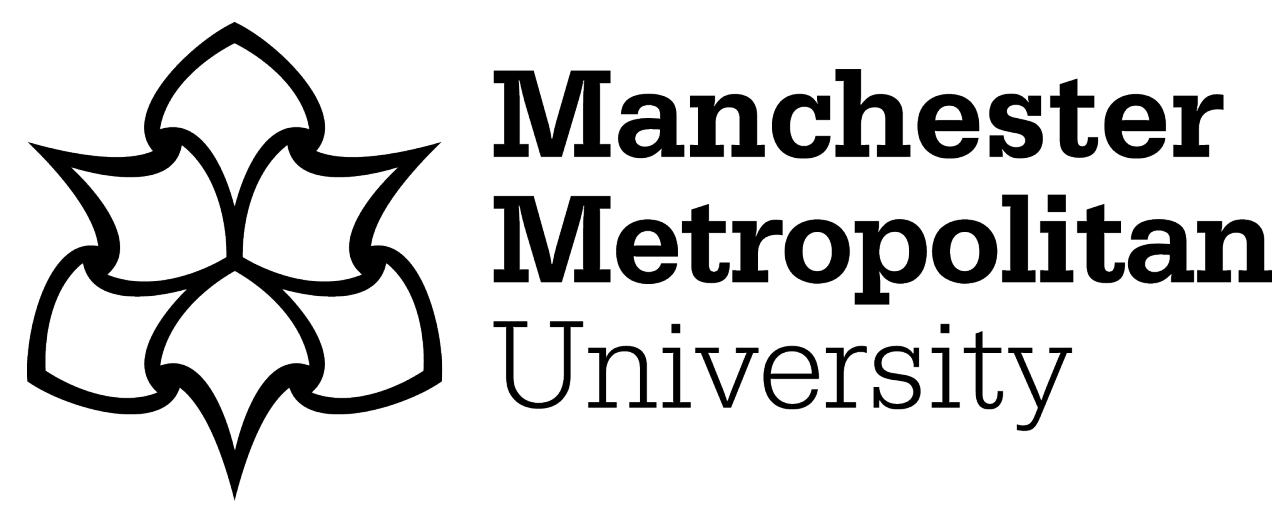

Weakley, J, Till, K, Read, D ORCID logoORCID: https://orcid.org/0000-00016367-0261, Leduc, C, Roe, G, Phibbs, P, Darrall-Jones, J and Jones, B (2018) Jump Training in Rugby Union Players : Barbell or Hexagonal Bar? Journal of Strength and Conditioning Research, 35 (3). pp. 754-761. ISSN 1064-8011

Downloaded from: https://e-space.mmu.ac.uk/625773/

Version: Accepted Version

Publisher: Wolters Kluwer

DOI: https://doi.org/10.1519/JSC.0000000000002742

Usage rights: Creative Commons: Attribution-Noncommercial 4.0

Please cite the published version 


\title{
Jump Training in Rugby Union Players: Barbell or Hexagonal Bar?
}

\author{
Jonathon J.S. Weakley, ${ }^{1,2}$ Kevin Till, ${ }^{1,2}$ Dale B. Read, ${ }^{1,2}$ Cedric Leduc, ${ }^{1}$ Gregory A. \\ B. Roe, ${ }^{1,3}$ Padraic J. Phibbs, ${ }^{1,2}$ Joshua Darrall-Jones, ${ }^{1,4}$ and Ben Jones ${ }^{1,2,5}$ \\ ${ }^{1}$ Institute for Sport, Physical Activity and Leisure, Leeds Beckett University, Leeds, West Yorkshire, United Kingdom; \\ ${ }^{2}$ Yorkshire Carnegie Rugby Union Club, Kirkstall Training Ground, Leeds Rugby Academy, Leeds, West Yorkshire, United \\ Kingdom; ${ }^{3}$ Bath Rugby, Bath, United Kingdom; ${ }^{4}$ Wasps Rugby, Coventry, United Kingdom; and ${ }^{5}$ The Rugby Football League, \\ Leeds, United Kingdom
}

\begin{abstract}
Weakley, JJS, Till, K, Read, DB, Leduc, C, Roe, GAB, Phibbs, $\mathrm{PJ}$, Darrall-Jones, J, and Jones, B. Jump training in rugby union players: barbell or hexagonal bar?. J Strength Cond Res $\mathrm{XX}(\mathrm{X})$ : 000-000, 2018-The countermovement jump (CMJ) is an exercise that can develop athletic performance. Using the conventional barbell (BAR) and hexagonal barbell (HEX) while jumping, the intensity can be increased. However, the bar that provides greater adaptations is unknown. Therefore, this study aimed to assess changes in loaded and unloaded CMJ with either a BAR or HEX across a 4-week mesocycle in rugby union players. Twenty-nine subjects were strength-matched and randomized into 2 groups. Subjects completed 3 sets of CMJ at $20 \%$ of 1 repetition maximum back squat, 3 times per week for 4 weeks, using either a BAR or HEX. Subjects completed an unloaded $\mathrm{CMJ}$ on a force plate before and after, whereas the highest peak concentric velocity during the jump squat was recorded in the first and last training sessions using a linear position transducer. Magnitude-based inferences assessed meaningful changes within- and between-groups. Possibly greater improvements in unloaded CMJ were found in the HEX group in jump height (effect size $\pm 90 \%$ confidence intervals: $0.27 \pm 0.27)$, relative peak $(0.21 \pm 0.23)$, and mean power $(0.32 \pm 0.36)$. In addition, likely to very likely greater improvements were observed in the HEX group in peak velocity $(0.33 \pm 0.27)$, relative mean power $(0.53 \pm 0.30)$, mean force $(0.47 \pm 0.27)$, and $100-m s$ impulse $(0.60 \pm 0.48)$. Similar raw changes in jump squat peak velocity occurred (0.20-0.25 $\left.\mathrm{m} \cdot \mathrm{s}^{-1}\right)$, despite the likely greater ES occurring with the BAR $(0.32 \pm 0.26)$. These results indicate that training with the HEX leads to superior unloaded $\mathrm{CMJ}$ adaptations. In addition, prac-
\end{abstract}

Address correspondence to Jonathon J.S. Weakley, j.j.weakley@ leedsbeckett.ac.uk. titioners should use either the HEX or BAR when aiming to enhance loaded jump ability.

KEY WoRDs force, power, velocity, impulse, jump squat, hexagonal barbell

\section{INTRODUCTION}

$\mathrm{T}$ he vertical countermovement jump (CMJ) is often incorporated within exercise routines and fitness testing batteries that are aimed at developing and assessing muscular power and athletic performance $(8,15)$. To increase the intensity of the CMJ, additional resistance can be added through external load (e.g., barbells, dumbbells, and weighted vests) $(26,27)$. The most common method of increasing external load during a $\mathrm{CMJ}$ is through the placement of a conventional barbell (BAR) over the posterior aspect of the shoulder (26). This variation of the $\mathrm{CMJ}$ is often referred to as the jump squat and has been recommended to be implemented within training programs for the development of lower-body power (7). Using this exercise within a resistance training program, large improvements in physical performance have been observed (8).

An alternative to the BAR jump squat is the use of a hexagonal barbell (HEX) with recent research suggesting this may be a safer and more effective alternative (26). The HEX allows individuals to stand within its frame and hold the external load at arms length (i.e., so that resistance is held approximately parallel to the hip or upper thigh, depending on make and subject arm length) (26,27). Recent research has demonstrated that when completing the jump squat with the HEX, athletes are able to produce greater force (effect size $[\mathrm{ES}]=0.47)$, power $(\mathrm{ES}=1.08)$, and velocity $(\mathrm{ES}=0.62)$ at the same relative intensity (e.g., $20 \%$ of 1 repetition maximum (1RM) of the back squat) compared with the BAR jump squat (26). These greater kinetic and kinematic outputs have been attributed to the external load being positioned closer to the athletes' center of mass that can result in favorable changes to the resistance moment 
TABle 1. Mean $\pm S D$ descriptive data of BAR and HEX groups.

\begin{tabular}{lcr}
\hline & BAR & HEX \\
\hline Age $(\mathrm{y})$ & $20.8 \pm 1.0$ & $21.4 \pm 1.6$ \\
Height $(\mathrm{cm})$ & $184.2 \pm 6.1$ & $186.1 \pm 6.6$ \\
Body mass $(\mathrm{kg})$ & $95.8 \pm 11.0$ & $94.9 \pm 9.7$ \\
3RM back squat $(\mathrm{kg})$ & $153.9 \pm 19.7$ & $154.8 \pm 20.3$ \\
Estimated 1RM back squat $(\mathrm{kg})$ & $165.5 \pm 21.2$ & $166.5 \pm 21.8$ \\
Prescribed jump squat load $(\mathrm{kg})$ & $33.1 \pm 4.2$ & $33.3 \pm 4.4$ \\
\hline
\end{tabular}

session across the 4-week mesocycle. Groups used either a conventional $20-\mathrm{kg}$ barbell or a $20-\mathrm{kg}$ hexagonal barbell that were both loaded to $20 \%$ of estimated 1RM back squat $(26,27)$. Subjects trained 3 times per week and were provided feedback on their peak concentric velocity after each repetition during all training sessions (22). Before and after the training program, all participants arms of individual joints (e.g., hips). Correspondingly, because of the increases in acute kinetic and kinematic outputs, it has been suggested that the HEX may promote superior physical adaptations $(21,26)$. However, changes from training with these different barbells have not been examined.

When performing the jump squat with a BAR or HEX, the load that optimizes power output has been subject to considerable debate $(10,26,27)$. When entire system mass (i.e., external load and body mass) is accounted for, it has been suggested that $0 \%$ of $1 \mathrm{RM}$ (i.e., body mass alone) maximizes power output (10). However, these conclusions were made despite the lack of statistical difference between peak power outputs at both 0 and $20 \%$ of 1RM (10). In addition, Turner et al. (27) found that peak power output in professional rugby union players in the HEX jump squat is optimized at a load of $10-20 \%$ 1RM. Nevertheless, although the load that maximizes acute peak power output in the BAR and HEX jump squat is established to be $0-20 \%$ of $1 \mathrm{RM}$ dependent on individual variations (e.g., body mass and training status) $(10,26,27)$, the effects of training at these intensities with these 2 different types of barbell has not been established.

Although the HEX has demonstrated greater acute force, power, and velocity production compared with the BAR at the same relative intensities (26), training adaptations using these different training apparatus have not been investigated. It is feasible that exposure to greater kinetic and kinematic outputs when training could promote greater changes in physical capacity. To this end, the aim of this study was to compare the effects of training with either a BAR or HEX on CMJ performance across a 4-week mesocyle within semiprofessional rugby union players. In addition, this study assessed changes in peak velocity in the BAR and HEX jump squat between the first and final training sessions of the 4-week mesocycle.

\section{Methods}

\section{Experimental Approach to the Problem}

To assess the effects of completing jump squats with either a BAR or HEX on jump performance, 29 semiprofessional rugby union players were randomly allocated into a BAR or HEX training group during a resistance training mesocycle (refer to Table 1). All subjects completed 3 sets of 3 jump squat repetitions at the beginning of each resistance training completed a body mass CMJ on a force platform that recorded kinetic and kinematic variables. In addition, each subject's highest peak concentric velocity value in the jump squat in the first and final (12th) training sessions was recorded.

\section{Subjects}

Twenty-nine male subjects (BAR group: 16 subjects; HEX group: 13 subjects), between the ages of 18 and 24, completed the training and testing protocols. Thirty-three semiprofessional rugby players were initially recruited to take part in this study. However, 4 subjects were not included during the final analysis because they did not attend $100 \%$ of the training sessions. All subjects had at least 2 years of resistance training experience $(3.6 \pm 1.1$ years) and were recruited from a British University and Colleges Super Rugby squad in the United Kingdom. The training and testing took place across the months of August and September (which is during the preseason period of the rugby union playing calendar). Recruited subjects confirmed that they did not have any current injuries, do not consume any medications or supplements that could influence performance and adaptations, and were not suffering from any diseases. Before the study, all subjects had completed a 6-week standardized preparatory phase where all resistance training exercises and intensities were specified. Furthermore, all subjects had previous experience of training that involved jump squats with both the BAR and HEX. Subjects were explained the design of the study, were provided an opportunity to ask questions, and then provided informed written consent, which they returned before participating in the study. All experimental procedures were approved by Leeds Beckett University's ethics committee, and written assent was provided by all subjects.

\section{Procedures}

Preceding the initial testing session, all subjects were provided 72 hours active rest and then were required to complete (a) an unloaded CMJ on a force platform (ForceDecks Model FD4000a; NMP Technologies Ltd., London, United Kingdom) and (b) a 3RM barbell back squat. Subjects were then strength-matched (using maximal back squat strength) and randomly assigned into 1 of 2 groups (i.e., BAR or HEX). During the following 4 weeks, all subjects completed $100 \%$ of the resistance $(3$ sessions per week; refer to Table 2 for resistance training sessions) 
and field training sessions ( 3 sessions per week). Each session was initiated with the subject completing a dynamic warm-up and then completing 3 sets of 3 jump squats using either the BAR or HEX. Previous research (22) has used the same protocols (i.e., number of sessions, sets, repetitions, and intensity) to assess the effect of jump squat training on physical development. The external load for each subject in both the BAR and HEX group was prescribed at $20 \%$ of estimated back squat 1RM and adjusted to the nearest whole kilogram (6). All subjects were required to be as "explosive and as forceful" as possible during all repetitions. After every repetition of the jump squat exercise, visual kinematic feedback of peak concentric velocity was provided by a GymAware (Kinetic Performance Technology, Canberra, Australia) linear position transducer to assist motivation and competitiveness (32). In the first and last (i.e., the 12th) resistance training sessions, the highest peak concentric velocity reported by the linear position transducer was recorded by the lead researcher. At the end of the 4-week mesocycle and 48 hours after the final training session, subjects completed another CMJ (using the same procedures that were completed during pretesting) on a force plate.

Countermovement Jump. Analysis of CMJ was completed before and after the training mesocycle using a force platform (ForceDecks Model FD4000a, NMP Technologies Ltd.), which sampled at a rate of $1,000 \mathrm{~Hz}$. All participants performed $3 \mathrm{CMJs}$ with feet placed approximately shoulder width apart and with hands placed on hips $(24,31)$. Participants lowered themselves to a selfselected depth and jumped as high as possible. Between each maximal exertion, at least 60 -second rest was provided $(24,31)$. We chose the following CMJ variables for analysis based on previously published between-day reliability statistics in a similar cohort (25). These were CMJ height (jump height), peak velocity, relative peak and mean power, mean power and force, and impulse at 100 ms. These variables were also selected because of their close relationship with physical performance $(14,33)$ and previous use in rugby union players $(24,30,31)$.

3RM Strength Assessment. Assessment of the 3RM back squat was chosen because this is regularly completed in rugby union players of a similar standard $(9,30,31)$ and is commonly used for exercise prescription $(29,31,32)$. In addition, all subjects within this cohort were familiar with the 3RM testing protocol. Players first completed a dynamic warm-up, which has previously been completed before maximal 3RM attempts (31). Maximal back squat strength was completed with a barbell (Eleiko Sport AB, Halmstad, Sweden) resting on the upper trapezium with participants grasping the bar with a pronated grip. Participants were required to lower 
themselves, so that the top of the thigh was observed (by the lead researcher) to be parallel with the floor, whereas the chest was required to remain upright. Heels were to remain in contact with the ground throughout the movement, and the participant was to return to the initial standing position.

Exercise Training Routine. Table 2 outlines the resistance training protocols that all subjects undertook across the 4 -week mesocycle. The resistance training exercises and sessions prescribed were part of the regular preseason training routine and were based on previous research by Randell et al. (22). There were 2 different training routines (session 1 and session 2) and these were completed in an alternating order (e.g., session 1 during resistance training session 1; session 2 during resistance training session 2), so that each individual session was completed 6 times. At the beginning of each resistance training workout, a dynamic warm-up would be undertaken followed by 3 sets of 3 repetitions of jump squat. Subjects within the BAR jump squat group completed the jump squat with a BAR that rested across the posterior aspect of the shoulder, whereas subjects within the HEX jump squat group held the HEX parallel to the hip/thigh. Both groups completed the same number of repetitions at the same intensity across all training sessions. Following on from the jump squat, a high-intensity ( $\sim 85-93 \%$ of $1 \mathrm{RM}$ back squat) bilateral lower-body movement was completed (refer to Table 2). During the back squat, depth was monitored by ensuring that all subjects touched a box, so that the upper thigh was parallel to the ground. In the HEX deadlift, the weight started on the ground and was raised until the participant was standing fully upright. Both movements were required to be completed with maximal intent. All other movements (e.g., 10-m accelerations and upper-body movements) were also completed with maximal intent but using methodology previously detailed within literature pertaining to resistance training in rugby union $(22,30,31)$. All field sessions involved a rugby union skill emphasis with all subjects taking part in each session.

Kinematic Feedback and Collection of Peak Concentric Velocity. Across the training mesocycle, subjects were provided feedback of peak concentric velocity during each repetition of the jump squat. All repetitions were recorded with a GymAware linear position transducer, which sampled at $50 \mathrm{~Hz}$. The optical encoder, which was placed directly below the BAR or HEX during the jump squat exercise, contains a retractable cord that was attached to the barbell during each set for each subject. Velocity and displacement were calculated from the rotation of a pulley system within the optical encoder on the movement of the barbell during the exercise (23). The encoder, which has previously been assessed as 


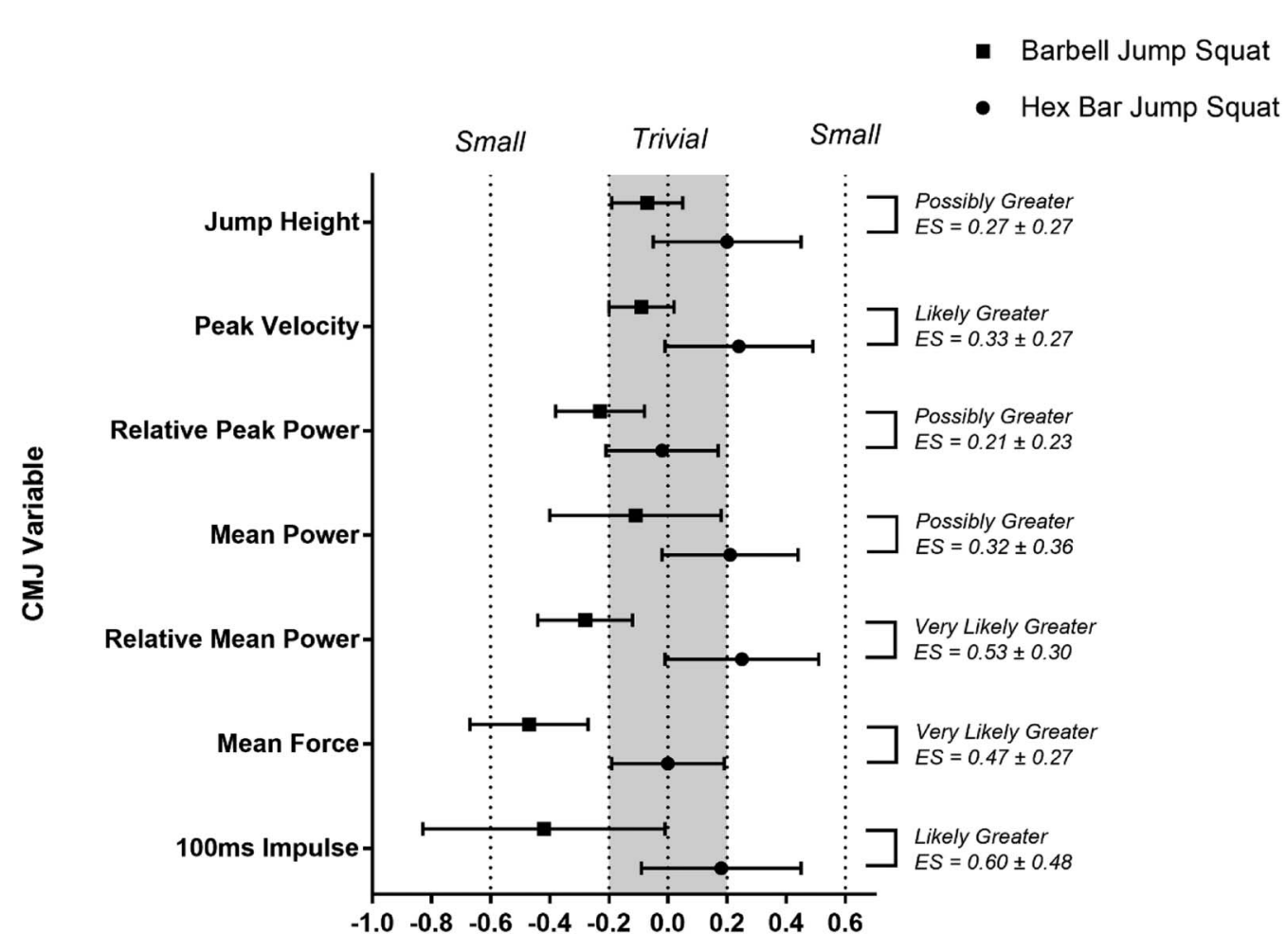

Standardised Effect on CMJ Variable

Figure 1. Standardized effect size $(\mathrm{ES})( \pm 90 \% \mathrm{Cl})$ change in countermovement jump variables and corresponding inference and ES of between-group differences.

highly valid for reporting of velocity, power, and force (3), provides approximately 1 electrical impulse every $3 \mathrm{~mm}$ of barbell displacement with each value time stamped with a 1-millisecond resolution. This velocity information was then transmitted to an iPad (iPad Pro; Apple, Inc., Cupertino, CA, USA), which was placed directly in front of subjects at standing eye level.

\section{Statistical Analyses}

Data are presented as either mean $\pm S D$ or percentage/ effect size (ES) $\pm 90 \%$ confidence intervals $(90 \% \mathrm{CIs})$ where specified. Before analysis, all data were log-transformed to reduce bias arising from nonuniformity error and then analyzed for practical significance using magnitude-based inferences (4). The chance of the CMJ variables or peak concentric velocity across the mesocycle being lower, similar, or greater than the smallest worthwhile change/ difference $(\mathrm{SWC} / \mathrm{D})$ (i.e., $0.2 \times$ between subject difference) was calculated using an online spreadsheet (11), with all between-group comparisons of effects being further analyzed using a separate spreadsheet (13). The probability that the magnitude of change was greater than the SWC/D was rated as $<0.5 \%$, almost certainly not; $0.5-5 \%$, very unlikely;
5-25\%, unlikely; 25-75\%, possibly; 75-95\%, likely; 95-99.5\%, very likely; and $>99.5 \%$, almost certainly (12). Where the $90 \%$ CI crossed both the upper and lower boundaries of the SWC (ES \pm 0.2$)$, the magnitude of change was described as unclear (4). Effect size thresholds were set at $<0.2$ (trivial), 0.2-0.6 (small), 0.6-1.2 (moderate), and 1.2-2.0 (large) (12).

\section{Results}

Pre- and post-CMJ values and corresponding within-group inferences are shown in Table 3. Standardized ES $( \pm 90 \%$ CI $)$ and inferences comparing between-group differences are presented in Figure 1. Individual subject and group mean $( \pm S D)$ peak concentric velocity values reported in session 1 and 12 of the 4-week mesocycle are presented in Figure 2. Mean peak concentric velocity in session 1 of the BAR jump squat was $2.28 \pm 0.14 \mathrm{~m} \cdot \mathrm{s}^{-1}$. By session 12 , this had increased to $2.49 \pm 0.17 \mathrm{~m} \cdot \mathrm{s}^{-1}(\mathrm{ES} \pm 90 \% \mathrm{CI}: 1.28 \pm$ 0.25). Mean peak concentric velocity in session 1 of the HEX jump squat was $2.55 \pm 0.20 \mathrm{~m} \cdot \mathrm{s}^{-1}$. By session 12 , this had increased to $2.78 \pm 0.23 \mathrm{~m} \cdot \mathrm{s}^{-1}$ (ES $\pm 90 \% \mathrm{CI}: 0.96 \pm$ $0.10)$. Between-group comparison of ES $( \pm 90 \% \mathrm{CI})$ changes shows likely greater improvements in the BAR condition $(0.32 \pm 0.26)$. 


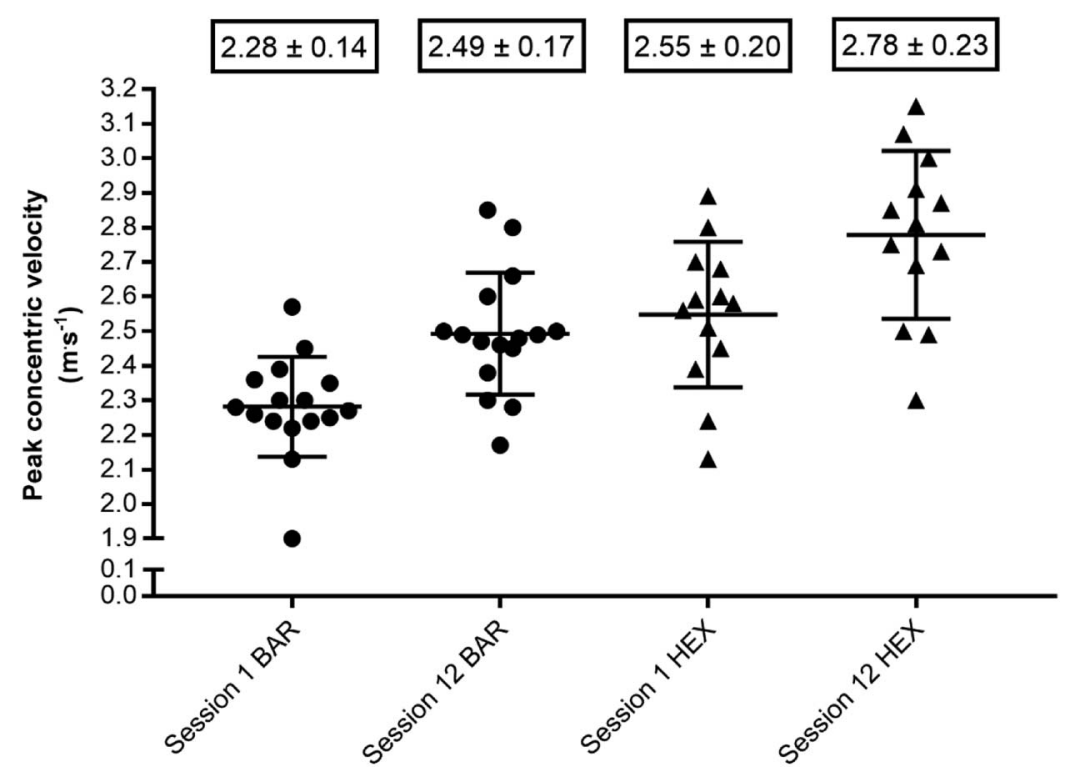

Figure 2. Individual and group mean $( \pm S D)$ changes in peak concentric velocity in sessions 1 and 12 .

\section{Discussion}

The aim of this study was to present and compare the effects of completing jump squat with either the BAR or HEX across a training mesocycle. After 4 weeks, the HEX group showed possibly greater improvements in CMJ height, relative peak power, and mean power. In addition, the HEX group showed likely greater improvements in peak velocity and impulse at $100 \mathrm{~ms}$, and very likely greater improvements in relative mean power and mean force. Conversely, the BAR group showed likely greater improvements in loaded jump squat peak velocity, despite similar raw changes, because of lower initial training velocities in the jump squat. These outcomes indicate that, while both forms of jump squat training promote improvements in peak velocity at $20 \%$ of $1 \mathrm{RM}$, the HEX jump squat may be a superior alternative to the BAR jump squat due to greater improvements in the unloaded CMJ power, velocity, and jump height. These findings suggest that performing HEX jump squats can promote greater adaptations in physical capacity.

Changes across the training mesocycle showed subjects within the HEX condition had possible improvements in jump height (ES $\pm 90 \%$ CI: $0.20 \pm 0.25)$ and peak velocity $(0.24 \pm 0.25)$. Conversely, the BAR condition showed very likely $(-0.07 \pm 0.12)$ and likely trivial $(-0.09 \pm 0.11)$ changes, respectively. These findings may partially be explained by acute differences in kinematic outputs when using the BAR and HEX for jump squat (26). Swinton et al. (26) have previously shown that when these different types of bar are loaded with $20 \%$ of $1 \mathrm{RM}$, the HEX allows for greater jump heights to be achieved. However, these differences were only shown to occur at $20 \%$ of $1 \mathrm{RM}$. These disparities were attributed to the significantly lower peak velocities that are produced when performing the jump squat with the BAR (compared with the HEX), and the close relationship this variable has with ballistic performance $(17,19)$. Consequently, it is possible that continued exposure to higher training velocities when using the HEX may have provided a greater stimulus for the development of jump height and peak velocity.

Although trivial changes in peak velocity and jump height occurred within the BAR condition, trivial to likely negative changes in mean $(\mathrm{ES} \pm 90 \% \mathrm{CI}:-0.11 \pm 0.29)$ and relative mean $(-0.28 \pm 0.16)$ power output were reported. Conversely, the HEX condition showed possible improvements in mean $(0.21 \pm 0.23)$ and relative mean $(0.25 \pm 0.26)$ power output. These differences may be explained by differences in changes in force-related variables (e.g., mean force and 100-ms impulse) (refer to Figure 1) (16). Within the BAR condition, very likely and likely negative changes in mean force and impulse were reported, respectively. Conversely, these variables remained relatively stable within the HEX condition. Consequently, it is theorized that reductions in force-related variables and maintenance of peak concentric velocity within the BAR condition may have caused possible decreases in power. However, it should also be noted that, in the BAR group, small negative changes were only reported in relative power measures (e.g., relative peak and mean concentric power), whereas mean power reported possibly trivial changes. Therefore, fluctuations in body mass could also be attributed to these outcomes. 
Irrespectively, subjects within the HEX condition showed possible small positive changes in both mean and relative mean power output production. Consequently, it is suggested that the HEX be used preferentially to promote the development of power output in athletes.

Within-group changes that were reported in force and impulse at $100 \mathrm{~ms}$ demonstrate an interesting aspect of this study. Although likely and possibly trivial changes in mean force (ES $\pm 90 \% \mathrm{CI}: 0.00 \pm 0.19)$ and impulse at $100 \mathrm{~ms}$ $(0.18 \pm 0.27)$ occurred within the HEX group, very likely $(-0.47 \pm 0.20)$ and likely $(-0.42 \pm 0.41)$ decreases occurred within the BAR group, respectively. These reported changes suggest that training with a BAR at $20 \%$ of $1 \mathrm{RM}$ did not promote positive adaptations in acceleration of body mass. This loss may be particularly pertinent during initial stages of the CMJ (i.e., the first $100 \mathrm{~ms}$ ). It is believed that these changes may have occurred because of the differences in bar placement during training. Previous research has stated that the placement of the BAR on the posterior aspect of the shoulder during the jump squat can limit forward inclination of the trunk at the bottom of the countermovement $(2,20,28)$ and cause larger moment arms around the knee and reduced recruitment of the hip extensor muscles (28). These changes are known to reduce jump height and power output (28). In addition, it has been stated that, using the HEX during the jump squat, unloaded jump performance is more closely resembled $(26,27)$. Consequently, these differences in jump technique and muscle recruitment may have impacted on force-generating adaptations. Considering this, the HEX may promote or maintain mean concentric and earlyphase force development (i.e., during the first $100 \mathrm{~ms}$ of a CMJ) to a greater extent when compared with the barbell jump squat.

When completing jump squat with $20 \%$ of $1 \mathrm{RM}$, almost certain improvements in peak velocity were observed in both groups across the mesocycle. Although similar raw changes were reported, greater effects $( \pm 90 \% \mathrm{CI})$ were observed in the BAR condition $(1.42 \pm 0.27$ vs. $0.96 \pm$ 0.10 ). This suggests that, irrespective of chosen apparatus, moderate to large improvements in peak velocity occur at the intensity that is trained $(1,18)$. This corroborates with Cormie et al. (8) who demonstrated that athletes show the largest adaptations at the intensities that are trained. However, these improvements in loaded jump squat performance may not have similar transference to body mass only performance (e.g., the HEX condition showed possible improvements in unloaded CMJ peak velocity, whereas the BAR condition showed likely trivial). This may occur for a number of reasons, but it is hypothesized that velocities completed during the HEX condition more closely replicated the velocities that were achieved when performing an unloaded jump $(26,27)$. In addition, by transitioning the barbell closer to the center of mass (i.e., during the HEX condition), athletes can more closely replicate their unloaded vertical jump technique (26).
Although this study is the first to examine the physical adaptations of using either the BAR or HEX when performing the jump squat, it is not without its limitations. First, any potential differences in on-field training were unable to be accounted for. Although all subjects within this study were from the same training squad and took part in the exact same training exercises, slight differences in rugby training loads cannot be dismissed. To counter this, the authors ensured that a range of playing positions were included within each condition, and that all sessions (i.e., resistance training and field) were completed. Second, because of the lack of a control group, we cannot definitively state that outcomes from these 2 different training methods are a result of the intervention or random error when assessing withingroup changes. However, as there were substantial differences between the 2 training groups, we can make inferences about the efficacy of the HEX over the BAR for improving $\mathrm{CMJ}$ variables. Third, the training routine that was completed was across a 4-week mesocycle. Although longer exposure to the training routine may have caused greater training effects, this mesocycle length was chosen because it mimics actual training practice and has ecological validity for the athletes involved $(5,22)$. Finally, the intensity selected for the HEX was derived from the back squat exercise. This methodology has previously been used to investigate the acute kinetic and kinematic outputs of completing the jump squat with both the BAR and HEX $(26,27)$. Moreover, it was decided necessary to control for intensity using the back squat because the HEX jump squat uses an explosive stretch-shortening cycle action (similar to the back squat and BAR jump squat), whereas the HEX deadlift starts from the floor without a preceding lowering phase.

In conclusion, the HEX jump squat seems to promote greater physical adaptation in the unloaded CMJ when compared with the BAR jump squat. These results may be of benefit for athletes aiming to improve velocity, power, and force characteristics. In addition, almost certain improvements in peak velocity occur in the jump squat, irrespective of the type of bar that subjects use when training at $20 \%$ of $1 \mathrm{RM}$ of back squat. However, the greater training velocities and similarities in movement between the HEX jump squat and the unloaded CMJ may encourage greater adaptations in unloaded performance. With these findings, the practitioner is advised to use the HEX when programming jump squat into a training mesocycle.

\section{Practical Applications}

The jump squat is a commonly used exercise that can promote lower-body power and can be completed with a range of different apparatus (e.g., barbells, dumbbells, and weighted vests). Findings from this study suggest that the HEX jump squat could be a superior alternative for the development of jump height, velocity, power, and force when compared with the BAR jump squat. Within this study, subjects completed 3 sets of 3 repetitions, with 2- to 
3-minute rest between each set at the beginning of each training session. In addition, subjects completed this exercise with relatively light loads (i.e., $20 \%$ of $1 \mathrm{RM}$ back squat) because this has been reported to maintain high concentric kinetic and kinematic outputs when performing loaded jumps. Consequently, it is suggested that practitioners use a similar loading protocol that includes the HEX bar.

\section{REFERENCES}

1. Aagaard, P, Simonsen, E, Trolle, M, Bangsbo, J, and Klausen, K. Specificity of training velocity and training load on gains in isokinetic knee joint strength. Acta Physiol 156: 123-129, 1996.

2. Argus, CK, Gill, ND, Keogh, JW, and Hopkins, WG. Assessing lower-body peak power in elite rugby-union players. J Strength Cond Res 25: 1616-1621, 2011.

3. Banyard, HG, Nosaka, K, Sato, K, and Haff, GG. Validity of various methods for determining velocity, force and power in the back squat. Int J Sports Physiol Perform 12: 1170-1176, 2017.

4. Batterham, AM and Hopkins, WG. Making meaningful inferences about magnitudes. Int J Sports Physiol Perform 1: 50-57, 2006.

5. Bompa, TO and Haff, GG. Traning cycles. In: Periodization: theory and methodology of training. Champaign, IL: Human Kinetics, 2009. pp. 229-234.

6. Caulfield, S. Resistance training exercises. In: Baechle, TR and Earle, RW, eds. Essentials of strength training and conditioning. Champaign, IL: Human Kinetics, 2008. pp. 396-399.

7. Cormie, P, McCaulley, GO, Triplett, NT, and McBride, JM. Optimal loading for maximal power output during lower-body resistance exercises. Med Sci Sports Exerc 39: 340-349, 2007.

8. Cormie, P, McGuigan, MR, and Newton, RU. Adaptations in athletic performance after ballistic power versus strength training. Med Sci Sports Exerc 42: 1582-1598, 2010.

9. Darrall-Jones, JD, Jones, B, and Till, K. Anthropometric and physical profiles of English academy rugby union players. $J$ Strength Cond Res 29: 2086-2096, 2015.

10. Dayne, AM, McBride, JM, Nuzzo, JL, Triplett, NT, Skinner, J, and Burr, A. Power output in the jump squat in adolescent male athletes. $J$ Strength Cond Res 25: 585-589, 2011.

11. Hopkins, W. Reliability from consecutive pairs of trials (excel spreadsheet). Sportsci 10: 50-53, 2006.

12. Hopkins, W, Marshall, S, Batterham, A, and Hanin, J. Progressive statistics for studies in sports medicine and exercise science. Med Sci Sports Exerc 41: 3, 2009.

13. Hopkins, WG. A spreadsheet for combining outcomes from several subject groups. Sportsci 10: 50-53, 2006.

14. Hori, N, Newton, RU, Andrews, WA, Kawamori, N, McGuigan, $\mathrm{MR}$, and Nosaka, K. Does performance of hang power clean differentiate performance of jumping, sprinting, and changing of direction? J Strength Cond Res 22: 412-418, 2008.

15. Laffaye, G, Wagner, PP, and Tombleson, TI. Countermovement jump height: Gender and sport-specific differences in the force-time variables. J Strength Cond Res 28: 1096-1105, 2014.

16. Linthorne, NP. Analysis of standing vertical jumps using a force platform. Am J Phys 69: 1198-1204, 2001.
17. Mann, JB, Ivey, PA, and Sayers, SP. Velocity-based training in football. Strength Cond J 37: 52-57, 2015.

18. McBride, JM, Triplett-McBride, T, Davie, A, and Newton, RU. The effect of heavy- vs. light-load jump squats on the development of strength, power, and speed. J Strength Cond Res 16: 75-82, 2002.

19. Moir, GL. Three different methods of calculating vertical jump height from force platform data in men and women. Meas Phys Educ Exerc Sci 12: 207-218, 2008.

20. Mundy, PD, Smith, NA, Lauder, MA, and Lake, JP. The effects of barbell load on countermovement vertical jump power and net impulse. J Sports Sci 35: 1-7, 2017.

21. Pareja-Blanco, F, Rodriguez-Rosell, D, Sanchez-Medina, L, Gorostiaga, EM, and Gonzalez-Badillo, JJ. Effect of movement velocity during resistance training on neuromuscular performance. Int J Sports Med 35: 916-924, 2014.

22. Randell, AD, Cronin, JB, Keogh, JW, Gill, ND, and Pedersen, MC. Effect of instantaneous performance feedback during 6 weeks of velocity-based resistance training on sport-specific performance tests. J Strength Cond Res 25: 87-93, 2011.

23. Randell, AD, Cronin, JB, Keogh, JW, Gill, ND, and Pedersen, MC. Reliability of performance velocity for jump squats under feedback and nonfeedback conditions. J Strength Cond Res 25: 3514-3518, 2011.

24. Roe, G, Till, K, Darrall-Jones, J, Phibbs, P, Weakley, J, Read, D, et al. Changes in markers of fatigue following a competitive match in elite academy rugby union players. S Afr J Sports Med 28: 1-4, 2016.

25. Roe, G, Darrall-Jones, J, Till, K, Phibbs, P, Read, D, Weakley, J, et al. Between-day reliability and sensitivity of common fatigue measures in rugby players. Int J Sports Physiol Perform 11: 581-586, 2016.

26. Swinton, PA, Stewart, AD, Lloyd, R, Agouris, I, and Keogh, JW. Effect of load positioning on the kinematics and kinetics of weighted vertical jumps. J Strength Cond Res 26: 906-913, 2012.

27. Turner, TS, Tobin, DP, and Delahunt, E. Optimal loading range for the development of peak power output in the hexagonal barbell jump squat. J Strength Cond Res 29: 1627-1632, 2015.

28. Vanrenterghem, J, Lees, A, and Clercq, DD. Effect of forward trunk inclination on joint power output in vertical jumping. J Strength Cond Res 22: 708-714, 2008.

29. Weakley, JJ, Till, K, Read, DB, Phibbs, PJ, Roe, G, Darrall-Jones, J, et al. The effects of superset configuration on kinetic, kinematic, and perceived exertion in the barbell bench press. J Strength Cond Res 2017. Epub ahead of print.

30. Weakley, JJ, Till, K, Darrall-Jones, J, Roe, GA, Phibbs, PJ, Read, D, et al. Strength and conditioning practices in adolescent rugby players: Relationship with changes in physical qualities. J Strength Cond Res 2017. Epub ahead of print.

31. Weakley, JJ, Till, K, Read, DB, Roe, GA, Darrall-Jones, J, Phibbs, PJ, et al. The effects of traditional, superset, and tri-set resistance training structures on perceived intensity and physiological responses. Euro J App Physiol 117: 1877-1889, 2017.

32. Weakley, JJ, Wilson, KM, Till, K, Read, DB, Darrall-Jones, J, Roe, $\mathrm{GA}$, et al. Visual feedback attenuates mean concentric barbel velocity loss, and improves motivation, competitiveness, and perceived workload in male adolescent athletes. $J$ Strength Cond Res 2017. Epub ahead of print.

33. Winter, EM, Abt, G, Brookes, FB, Challis, JH, Fowler, NE, Knudson, DV, et al. Misuse of "power" and other mechanical terms in sport and exercise science research. J Strength Cond Res 30: 292-300, 2016. 